OPEN ACCESS

Edited by:

Ming Yang

Sichuan University, China

Reviewed by:

Tomasz Kostka,

Medical University of Lodz, Poland

Irma Ruslina Defi

Dr. Hasan Sadikin General

Hospital, Indonesia

${ }^{*}$ Correspondence:

Jian-Ping Cai

caijp61@vip.sina.com

Specialty section: This article was submitted to

Geriatric Medicine,

a section of the journa

Frontiers in Medicine

Received: 17 November 2021

Accepted: 26 January 2022

Published: 18 February 2022

Citation:

Jiang S, Cui J, Zhang L-Q, Liu Z, Zhang Y, Shi Y and Cai J-P (2022)

Role of a Urinary Biomarker in the

Common Mechanism of Physical Performance and Cognitive Function.

Front. Med. 9:816822.

doi: 10.3389/fmed.2022.816822

\section{Role of a Urinary Biomarker in the Common Mechanism of Physical Performance and Cognitive Function}

\author{
Shan Jiang ${ }^{1,2,3}$, Ju Cui ${ }^{1,2,3}$, Li-Qun Zhang ${ }^{1,2,3}$, Zhen Liu ${ }^{1,2,3}$, Yan Zhang ${ }^{1,2,3}$, Yuan Shi ${ }^{4}$ and \\ Jian-Ping Cai ${ }^{1,2,3 *}$ \\ ${ }^{1}$ The Key Laboratory of Geriatrics, Beijing Institute of Geriatrics, Beijing Hospital, Beijing, China, ${ }^{2}$ National Center of \\ Gerontology, National Health Commission, Beijing, China, ${ }^{3}$ Institute of Geriatric Medicine, Chinese Academy of Medical \\ Sciences, Beijing, China, ${ }^{4}$ Department of Geriatric Medicine, Longtan Community Health Service Center, Beijing, China
}

Introduction: Healthy aging is described as a process of developing and maintaining intrinsic abilities, including physical and cognitive functions. Although oxidative stress is a common mechanism shared by loss of muscle strength and dementia, its relationship with decreased physical performance and cognitive impairment remains unclear. We aimed to investigate the role of urinary 8-oxo-7, 8-dihydroguanosine (8-oxoGsn), a biomarker of oxidative damage to RNA, in physical and cognitive decline.

Methods: The study followed a cross-sectional design and recruited 40-94-year-old inhabitants of Beijing, China (471 men and 881 women). The physical performance of the participants was assessed using handgrip strength, walking speed, and the repeated chair stand test. The cognitive function was assessed using the Montreal Cognitive Assessment (MoCA) 5-min protocol. Urinary 8-oxoGsn levels were measured for all participants.

Results: Participants with high urinary 8-oxoGsn levels were more likely to have low grip strength, slow walking speed, poor performance in the repeated chair stand test, and low scores on the MoCA 5-min protocol (odds ratio [OR] 3.43, 95\% confidence interval [Cl]: 1.52-7.76; OR 1.71, 95\% Cl: 1.16-2.53; OR 2.06, 95\% Cl: 0.92-4.63; OR 1.75, 95\% Cl: 1.18-2.58), after adjusting for age, sex, smoking habits, alcohol consumption, hypertension, diabetes, cerebro-cardiovascular disease, and chronic kidney disease.

Conclusion: Elevated levels of oxidative stress are independently associated with cognitive and physical impairment. Thus, these results can help in the early identification and development of strategies for the prevention and treatment of intrinsic capacity decline.

Keywords: physical performance, cognitive function, oxidative stress, urinary 8-oxo-7, 8-dihydroguanosine, RNA oxidation

\section{INTRODUCTION}

Healthy aging, as described in the World Report on Aging and Health, developed by the World Health Organization, is the process of developing and maintaining intrinsic capacity, which includes all the physical and cognitive functions of an individual (1). Preventing the decline in physical and cognitive abilities is important for maintaining independence and promoting social 
participation. Further, researchers have indicated that the development of cognitive impairment and physical disability share many common pathophysiological mechanisms (2), and it is important to explore the common pathophysiological mechanisms and biomarkers of both. These results provide an attractive target for evaluating and developing intervention strategies of intrinsic capacity.

Oxidative stress is defined as an imbalance between the release of pro-oxidants and antioxidant defenses in favor of pro-oxidants, which can damage cell structures, leading to health problems. Oxidative stress has been postulated to be an underlying mechanism for the loss of muscle strength. From a biological perspective, with advancing age, the ability of muscle cells to process increased accumulation of reactive oxygen and nitrogen species (ROS/RNS) is compromised, leading to increased oxidative stress responses that impair cellular homeostasis and ultimately the loss of muscle strength and functional decline (3). Previous clinical studies have shown that increased levels of oxidative stress markers are associated with decreased muscle strength (4), sarcopenia (5), and frailty (6). In addition to muscle strength, oxidative stress plays an important role in the progression of Alzheimer's disease (AD) (7). Although the etiology and pathogenesis of AD are not fully understood, oxidative damage is a crucial component of $\mathrm{AD}$. The brain is highly susceptible to oxidative imbalance due to its high energy demand, high oxygen consumption, and relative deficiency of antioxidant enzymes, which directly affect the synaptic activity and neurotransmission in neurons, leading to cognitive impairment (8). In patients with $\mathrm{AD}$ and mild cognitive impairment, researchers have found elevated oxidative stress markers in brain tissue or peripheral blood and decreased total antioxidant capacity (9-11). Therefore, the role of oxidative stress in the development of physical and cognitive impairment should be analyzed.

Measurement of ROS is difficult because their half-lives are usually very short; therefore, the evaluation of oxidative stress in biological fluids is conducted by quantifying oxidative stress biomarkers. Previous studies have shown that oxidative stress can damage nucleic acids, lipids, and proteins. In addition, DNA and RNA precursor nucleotides are equally damaged by oxidation. In this regard, 8-oxo-7, 8-dihydro-2'-deoxyguanosine (8-oxodGsn) and 8-oxo-7, 8-dihydroguanosine (8-oxoGsn) are the two main nucleic acid oxidative adducts derived from DNA and RNA, respectively (12). These lesions are caused by oxidative damage at the guanine C-8 position, which, if left unrepaired, can result in G-to-T transversion events (13). However, among these, the most recurring is 8-oxoGsn. Previous studies have shown that RNA is more susceptible to oxidative damage than DNA because of the following reasons: (1) RNA is a single-stranded nucleic acid and is easily exposed to ROS; (2) RNA is less protected by proteins than DNA; (3) the active repair mechanism of oxidized RNA has not been identified; and (4) cytoplasmic RNAs are near the mitochondria, where many ROS are produced. Indeed, oxidative damage to RNA is more common than oxidative damage to DNA in humans $(14,15)$. Regarding this, 8 -oxoGsn is the most detectable and representative RNA oxidative damage product in urine and an indicator of oxidative stress level in vivo.
Although the development of muscle strength decline and dementia share common mechanisms, previous studies on oxidative stress and cognitive and muscle strength have usually been studied independently. In addition, blood tests were commonly used as indicators of oxidative stress. Urine samples are easier to obtain, and urinary 8-oxoGsn is stable and easy to store. However, its relationship with physical and cognitive decline in population-based studies has not yet been determined. In this study, we selected commonly used objective indicators of physical function, namely, grip strength, walking speed, and the repeated chair stand test, as well as the Montreal Cognitive Assessment 5-min protocol (MoCA 5min protocol) as a cognitive assessment tool, and aimed to examine the cross-sectional associations of urinary 8-oxoGsn and physical and cognitive decline to provide a basis for evaluating intrinsic capacity and targeted interventions from a pathophysiological perspective.

\section{MATERIALS AND METHODS}

\section{Participants}

All participants were recruited from the first wave of the China Aging Longitudinal Study (CALS), an ongoing cohort study that aims to explore health and aging trends in China. Beijing is one of the sub-centers of the CALS program. This study included community-dwelling adults who were conveniently sampled from the population of Beijing. The participants were recruited through advertisements in health examination centers and communities between December 2019 and December 2020. The eligibility criteria were as follows: (1) age $>40$ years and signed informed consent form; (2) no mental disorders; and (3) no history of alcohol or drug abuse. The exclusion criteria included acute medical treatment or hospitalization within the first 3 months of measurement; the presence of severe diseases, including acute heart, liver, or kidney disease, and respiratory failure; inability to walk independently; and previously diagnosed dementia.

\section{Sociodemographic Variables}

Trained personnel recorded demographic data during face-toface interviews. Health characteristics included body mass index (BMI), chronic diseases, and lifestyle. Height and body mass were measured using a digital scale. BMI $\left(\mathrm{kg} / \mathrm{m}^{2}\right)$ was calculated as body mass divided by height squared. Chronic diseases included hypertension, diabetes, cerebro-cardiovascular disease, and chronic kidney disease. Smoking habits were categorized as current or never/former smoker, and alcohol consumption was categorized as current or never/former drinker.

\section{Physical Performance}

Participants were asked to safely complete the grip strength, walking speed, and repeated chair stand tests while the examiners recorded the results. (1) Grip strength was measured using a grip dynamometer (EH101; Camry, Zhongshan, China). During the measurement, the participants stood upright, with their feet naturally separated and their arms relaxed, with full elbow extension. They were instructed to grip the handle as hard as 
possible. Further, the maximum values obtained from the two tests using the dominant hand were included in the analyses. (2) Walking speed: The participants were asked to walk at their usual pace along a 6-meter course. Additionally, although they were allowed to use a walking aid (a cane, walker, or other walking aid) if necessary, no assistance was provided by another person. The time required to complete the entire distance was recorded in seconds. (3) Repeated chair stand test: The participants were asked to stand from a sitting position without using their arms and to sit down again five times, as quickly as possible, with their arms folded across their chest. The time taken for rising from the chair to standing at the end of the fifth stand was taken and recorded. Cut-offs for maximal grip strength $(<28 \mathrm{~kg}$ for men and $<18 \mathrm{~kg}$ for women), walking speed $(<1.0 \mathrm{~m} / \mathrm{s})$, and repeated chair stand test $(\geq 12 \mathrm{~s})$ were based on the Asian Working Group for Sarcopenia (AWGS) 2019 guidelines (16).

\section{Cognitive Function}

The MoCA has been recommended as a clinical screening instrument for cognitive impairment; however, it is a relatively long test $(\sim 15$ mins $)$. In 2005 , the National Institute of Neurological Disorders and Stroke-Canadian Stroke Network Harmonization Working Group recommended a very brief protocol ( $\sim 5$ mins), which extracted four subtests from the MoCA (17). The MoCA 5-min protocol was designed to serve as a tool (for bedside screening and busy clinics) that can be used over the telephone and to support large epidemiological and clinical studies.

The MoCA 5-min protocol, which comprises four subtests, assesses five different cognitive domains as follows: attention, verbal learning, memory, executive functions/language, and orientation. In this study, the MoCA 5-min protocol was derived from the Hong Kong version of the MoCA (18). First, simple auditory attention span (also known as immediate memory) was measured using a five-word-learning trial, and the number of words recalled was recorded. Second, the participants were asked to recall as many names of animals as possible in $1 \mathrm{~min}$. While phonemic fluency was proposed in the English version, the Chinese language is based on a non-alphabetic system; therefore, a semantic fluency task (names of animals) was adopted in the study. Third, different weights were given to free and cued recalls, which reflect different types of memory failure (encoding vs. retrieval deficits). The total score of the MoCA 5-min protocol is 30 points; a raw score of $<25$ in welleducated persons (education $>12$ years) or a raw score of $<23$ in less-educated persons (education $\leq 12$ years) was defined as impaired global cognition (19). Table 1 provides a description of the MoCA 5-min protocol. All participants underwent a face-to-face cognitive assessment administered by trained interviewers.

\section{Oxidative Stress Biomarker}

To prepare the urine samples, the frozen urine was thawed, incubated at $37^{\circ} \mathrm{C}$ for 5 mins, and centrifuged at 7,500 $\times$ g for 5 mins at $4^{\circ} \mathrm{C}$. To each $200-\mu \mathrm{L}$ aliquot of the supernatant, $200 \mu \mathrm{L}$ of working solution ( $70 \%$ methanol, $30 \%$ water with $0.1 \%$ formic acid, and 5-mmol/L ammonium acetate) and $10 \mu \mathrm{L}\left[{ }^{15} \mathrm{~N}_{2}^{13} \mathrm{C}_{1}\right]$ of 8 -oxoGsn were used as an internal standard $(240 \rho \mathrm{g} / \mathrm{L})$. The
TABLE 1 | Description of MoCA 5-min protocol.

\begin{tabular}{|c|c|c|c|c|}
\hline $\begin{array}{l}\text { Cognitive } \\
\text { domain }\end{array}$ & Item & Description & Scoring & $\begin{array}{l}\text { Maximum } \\
\text { score }\end{array}$ \\
\hline Attention & 1 & $\begin{array}{l}\text { Immediate } \\
\text { recall of } 5 \\
\text { words }\end{array}$ & $\begin{array}{l}1 \text { point for each word } \\
\text { correctly recalled in the } \\
\text { first trial }\end{array}$ & 5 \\
\hline $\begin{array}{l}\text { Executive } \\
\text { function/language }\end{array}$ & 2 & $\begin{array}{l}\text { 1-min verbal } \\
\text { fluency } \\
\text { (animal } \\
\text { category) }\end{array}$ & $\begin{array}{l}0.5 \text { point for each } \\
\text { correct output }\end{array}$ & 9 \\
\hline Orientation & 3 & $\begin{array}{l}\text { 6-item date } \\
\text { and } \\
\text { geographic } \\
\text { orientation }\end{array}$ & $\begin{array}{l}1 \text { point for each correct } \\
\text { response }\end{array}$ & 6 \\
\hline \multirow[t]{2}{*}{ Memory } & 4 & $\begin{array}{l}\text { Delayed recall } \\
\text { and } \\
\text { recognition of } \\
5 \text { words } \\
\text { learned in } \\
\text { item } 1\end{array}$ & $\begin{array}{l}2 \text { points for each of the } \\
\text { words spontaneously } \\
\text { recalled; } 1 \text { point for } \\
\text { each word by cued } \\
\text { recall or recognition but } \\
\text { not spontaneously } \\
\text { recalled }\end{array}$ & 10 \\
\hline & & & Total & 30 \\
\hline
\end{tabular}

mixture was incubated at $37^{\circ} \mathrm{C}$ for 10 mins and subsequently centrifuged at $12,000 \times \mathrm{g}$ for $15 \mathrm{mins}$ at $4^{\circ} \mathrm{C}$. Finally, $5 \mu \mathrm{L}$ of the supernatant was injected for modified ultraperformance liquid chromatography and mass spectrometry analysis. Considering the variability in urinary volumes and significant differences in the renal glomerular function, the concentrations of each of the analytes were normalized relative to the amount of creatinine, and 8-oxoGsn was expressed in $\mu \mathrm{mol} / \mathrm{mol}$.

\section{Statistical Methods}

Urinary 8-oxoGsn was recorded using the 33rd and 66th percentiles as reference. Participants were categorized according to the tertiles of urinary 8-oxoGsn into the lowest, middle, and highest tertile groups. The chi-square statistic was used to test the association of urinary 8-oxoGsn with sex, chronic diseases (hypertension, diabetes, cerebro-cardiovascular disease, and chronic kidney disease), smoking habits, and alcohol consumption. Analysis of variance was used to examine the association of urinary 8-oxoGsn tertiles with selected continuous variables. The relationships between the variables were calculated using Pearson's product-moment correlations.

The following possible confounding factors were considered regarding the association between oxidative stress and physical and cognitive functions: (1) variables that were significantly different among the urinary 8-oxoGsn tertile groups and previously reported to be related to physical and cognitive functions (age, sex, smoking habits, alcohol consumption, hypertension, diabetes, and cerebro-cardiovascular disease), and (2) variables that had no significant difference among the diagnostic groups in this study but were reported to be related to physical and cognitive functions (chronic kidney disease), which were adjusted as covariates. 
Multiple linear regression analysis was used to assess the association of physical performance and cognitive function (dependent variables) with tertiles of urinary 8-oxoGsn (independent variables) after adjusting for confounders. The lowest tertile was used as a reference. Logistic regression analysis was performed to examine the correlation between impaired physical and cognitive function (dependent variables) and tertiles of urinary 8-oxoGsn (independent variables) after adjusting for confounders. All analyses were performed using SPSS software (version 25.0; SPSS, Inc, Chicago, IL). Statistical significance was set at $p<0.05$.

\section{RESULTS}

We studied 1,352 healthy participants (471 men [34.8\%] and 881 women [65.2\%]). The mean age of participants was 62.9 years (range: 40-94 years). Table 2 shows the health characteristics and cognitive and physical function parameters of the participants according to the tertiles of urinary 8-oxoGsn. Participants in the highest tertile of urinary 8-oxoGsn were, on average, older than those in the middle- and lowest tertiles. There was no significant difference in BMI among the three groups. High prevalence rates of hypertension, diabetes, and cerebrocardiovascular disease were observed in the highest tertile of urinary 8-oxoGsn. Smoking and alcohol consumption habits were significantly different among the three groups. Regarding physical performance, participants in the highest urinary 8oxoGsn tertile had a lower grip strength, slower walking speed, and required more time for completing the repeated chair stand test than those in the middle- and lowest tertile groups. In terms of cognitive function, the scores on the MoCA 5-min protocol were significantly lower in the highest tertile group than in the other two groups.

Pearson correlation coefficients were used to examine the relationship of urinary 8 -oxoGsn levels with physical performance and cognitive function parameters. Significant negative associations were found between the urinary 8-oxoGsn level and grip strength, walking speed, and the MoCA 5-min protocol score $(r=-0.41,-0.29$, and -0.21 , respectively; $p$ $<0.001$ ), whereas the urinary 8 -oxoGsn level was positively associated with the results of the repeated chair stand test $(r=0.35, p<0.001)$. The levels of urinary 8-oxoGsn in participants with different physical and cognitive functional statuses showed significant differences. The urinary 8-oxoGsn levels were significantly higher in the participants with low grip strength (men: $<28 \mathrm{~kg}$, women: $<18 \mathrm{~kg}$ ), slow walking speed $(<1$ $\mathrm{m} / \mathrm{s}$ ), slowly repeated chair stand test ( $\geq 12 \mathrm{secs}$ ), and impaired cognitive function (MoCA 5-min protocol: $<25$ ) than in those with normal values for these parameters $(2.72 \pm 0.87$ vs. 1.73 $\pm 0.76 \mu \mathrm{mol} / \mathrm{mol} ; 2.30 \pm 0.86$ vs. $1.68 \pm 0.73 \mu \mathrm{mol} / \mathrm{mol} ; 2.79$ \pm 0.78 vs. $1.70 \pm 0.74 \mu \mathrm{mol} / \mathrm{mol} ; 1.95 \pm 0.84$ vs. $1.63 \pm 0.73$ $\mu \mathrm{mol} / \mathrm{mol}, p<0.05)$. Therefore, higher urinary 8 -oxoGsn levels were found to be associated with poor physical performance and worse cognitive function.

After adjusting for confounders, the multiple linear regression method revealed that compared with the participants in the
TABLE 2 | Sociodemographic characteristics and cognitive and physical function parameters of the participants, according to tertile of urinary 8-oxoGsn.

\begin{tabular}{|c|c|c|c|c|}
\hline \multirow[t]{2}{*}{ Characteristics } & \multicolumn{4}{|c|}{ Urinary 8-oxoGsn } \\
\hline & $\begin{array}{l}\text { Lowest tertile, } \\
\qquad n=445\end{array}$ & $\begin{array}{l}\text { Middle tertile, } \\
\quad n=448\end{array}$ & $\begin{array}{l}\text { Highest tertile, } \\
\quad n=459\end{array}$ & $P$ \\
\hline $\begin{array}{l}\text { Urinary 8-oxoGsn } \\
(\mu \mathrm{mol} / \mathrm{mol})\end{array}$ & $1.0(0.2)$ & $1.6(0.2)$ & $2.8(0.6)$ & \\
\hline Age (years) & $56.2(8.6)$ & $61.4(10.5)$ & 70.6 (11.6) & $<0.001$ \\
\hline Females & $284(63.8)$ & $284(63.4)$ & $313(68.2)$ & $<0.001$ \\
\hline Education (years) & $14.2(2.8)$ & $13.7(3.0)$ & $13.2(2.9)$ & $<0.001$ \\
\hline \multicolumn{5}{|l|}{$\begin{array}{l}\text { Health } \\
\text { characteristics }\end{array}$} \\
\hline $\mathrm{BMI}\left(\mathrm{kg} / \mathrm{m}^{2}\right)$ & $24.4(3.1)$ & $24.6(3.7)$ & 24.5 (3.5) & 0.757 \\
\hline Hypertension & $128(28.8)$ & $169(37.7)$ & $210(45.7)$ & $<0.001$ \\
\hline Diabetes & $46(10.3)$ & $78(17.4)$ & $102(22.2)$ & $<0.001$ \\
\hline $\begin{array}{l}\text { Cerebro- } \\
\text { cardiovascular } \\
\text { disease }\end{array}$ & 101 (11.0) & $110(16.0)$ & $162(20.5)$ & $<0.001$ \\
\hline $\begin{array}{l}\text { Chronic kidney } \\
\text { disease }\end{array}$ & $12(3.1)$ & $15(3.8)$ & $20(4.9)$ & 0.387 \\
\hline \multicolumn{5}{|l|}{ Lifestyles } \\
\hline Smoking habit & $67(15.1)$ & 49 (10.9) & $80(17.4)$ & 0.019 \\
\hline $\begin{array}{l}\text { Alcohol } \\
\text { consumption }\end{array}$ & 89 (20.0) & $80(17.8)$ & $63(13.7)$ & 0.039 \\
\hline \multicolumn{5}{|l|}{$\begin{array}{l}\text { Physical } \\
\text { performance }\end{array}$} \\
\hline Grip strength (kg) & 33.0 (8.8) & 30.8 (8.6) & $25.1(7.7)$ & $<0.001$ \\
\hline $\begin{array}{l}\text { Walking speed } \\
(\mathrm{m} / \mathrm{s})\end{array}$ & $1.2(0.2)$ & $1.2(0.2)$ & $1.0(0.3)$ & $<0.001$ \\
\hline $\begin{array}{l}\text { Repeated chair } \\
\text { stands test (s) }\end{array}$ & $7.0(1.8)$ & $7.6(2.1)$ & $10.9(2.2)$ & $<0.001$ \\
\hline \multicolumn{5}{|l|}{$\begin{array}{l}\text { Cognitive } \\
\text { function }\end{array}$} \\
\hline $\begin{array}{l}\text { MoCA 5-min } \\
\text { protocol, points }\end{array}$ & $28.5(2.1)$ & $27.0(2.4)$ & $26.2(3.1)$ & $<0.001$ \\
\hline
\end{tabular}

Variables are expressed as mean (SD) or $n$ (\%) unless stated. 8-oxoGsn, 8-oxo-7, 8-dihydroguanosine; BMI, body mass index; MoCA, Montreal cognitive assessment.

lowest tertile of urinary 8-oxoGsn, those in the highest tertile of urinary 8-oxoGsn had an average decrease in grip strength of $2.55 \mathrm{~kg}$ (beta:-2.55, 95\% confidence interval [CI]:-3.45 $-1.65)$, reduction in walking speed of 0.08 secs (beta: $-0.08,95 \%$ CI:-0.12 to -0.05$)$, prolonged time of $2.03 \mathrm{sec}$ in the repeated chair stand test (beta: 2.03, 95\% CI: 0.97-3.03), and reduction in score on the MoCA 5 -min protocol by 0.4 (beta: $-0.40,95 \%$ CI: -0.86 to -0.07$)$. In the logistic regression model adjusted for confounders, the participants in the highest tertile of urinary 8oxoGsn were 3.43 times more likely to have low grip strength (men: $<28 \mathrm{~kg}$, women: $<18 \mathrm{~kg}$ ) than those in the lowest tertile (OR 3.43, 95\% CI: 1.52-7.76). The participants in the highest tertile of urinary 8-oxoGsn showed a significantly higher risk of impairment in walking speed $(<1 \mathrm{~m} / \mathrm{s}$ ) (OR 1.71, 95\% CI: 1.16-2.53) and poor performance in the repeated chair stand test ( $\geq 12$ secs) (OR 2.06, 95\% CI: 0.92-4.63) than those in the lowest tertile. In addition, the participants in the highest 

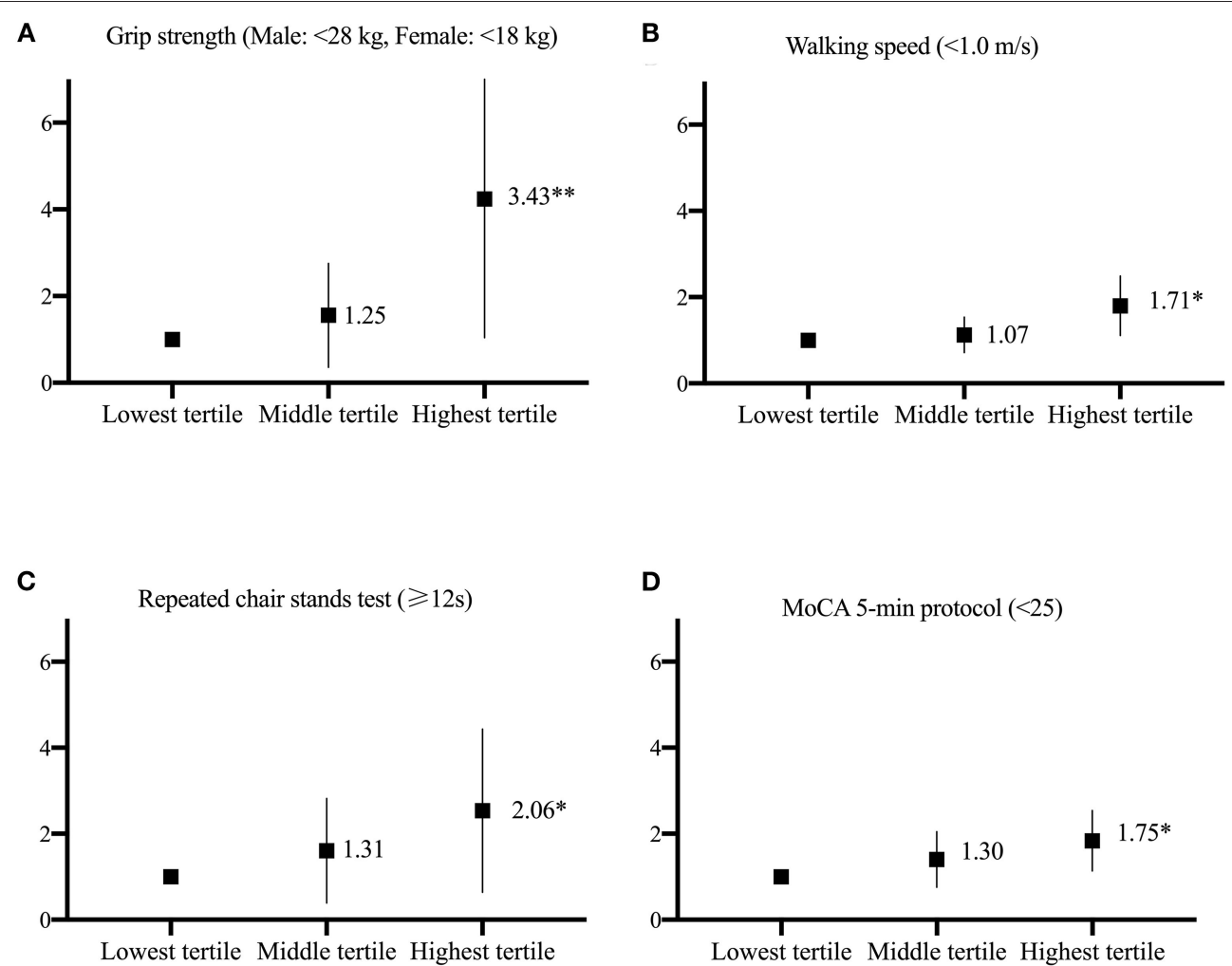

FIGURE 1 | The relationship between the different tertiles of urinary 8-oxoGsn and physical and cognitive function is represented (A-D). Odds ratio and 95\% CI derived from logistic regression models after adjusting for age, sex, smoking habits, alcohol consumption, hypertension, diabetes, cerebrocardiovascular disease, and chronic kidney disease. The lowest tertile of urinary 8-oxoGsn was used as the reference group ${ }^{*} p<0.05,{ }^{\star *} p<0.001$.

tertile of urinary 8-oxoGsn were 1.75 times more likely to have cognitive impairment (MoCA 5-min protocol $<25$ ) than those in the lowest tertile (OR 1.75, 95\% CI: 1.18-2.58). The relationship between the different tertiles of urinary 8-oxoGsn and physical and cognitive impairment is shown in Figure 1.

Previous studies have shown that 8-oxoGsn and physical and cognitive function are age-related. We plotted bar graphs showing the parameters of physical and cognitive function in the three 8-oxoGsn groups in different age categories (Supplementary Materials), further indicating that the relationship between 8 -oxoGsn and physical and cognitive function is age-independent.

\section{DISCUSSION}

Our cross-sectional study, which involved more than 1,000 community-dwelling middle-aged or old-aged adults, revealed that increased urinary 8-oxoGsn levels are independently associated with poor physical performance and cognitive impairment; and that a high level of oxidative stress is related to cognitive and physical decline.

Oxidative Stress and Physical Performance Decline in muscle function is caused by a decrease in muscle mass and quality. Oxidative stress is supposedly a common determinant of the loss of both muscle mass and quality. This can be explained by the following mechanisms: oxidative stress has the potential to reduce muscle mass by increasing proteolysis and decreasing muscle protein synthesis (20). The mechanisms involved in oxidative stress-mediated muscle quality impairment include reduction in acetylcholine release at the synaptic cleft, leading to a failure of the sarcolemma to produce action potentials (21). This alters the morphology of the motor neuron and neuromuscular junction and ultimately resulting in loss of innervation and reduced number of fibers (21); excitationcontraction coupling failure stemming from redox-induced disruption of calcium release from the sarcoplasmic reticulum (22); and redox modifications to actin and myosin structures that reduce the cross-bridge cycling within the myofibrillar apparatus (23).

Sarcopenia is a clinical sign characterized by diminished skeletal muscle mass and poor muscle function, resulting in frailty and disability (24). Previous studies have shown that elevated oxidative stress is closely linked to age-associated sarcopenia (4). A systematic review showed that frailty and prefrailty are associated with higher peripheral levels of oxidative stress biomarkers and lower antioxidant parameters (25). A cross-sectional study of 672 women aged $\geq 65$ years reported an independent association between oxidative protein damage and low grip strength, suggesting that oxidative stress might 
contribute to the loss of muscle strength (26). Oxidative stress has been implicated in sarcopenia and loss of muscle strength with aging; however, the relationship between oxidative stress and decrease in multiple indicators of physical performance has not been sufficiently researched. Our previous research showed that urinary 8-oxoGsn, a recognized biomarker of RNA oxidation, was independently associated with frailty in elderly patients with cardiovascular disease and community-dwelling older adults $(27,28)$. However, there are limited studies on the correlation between this biomarker and indicators of physical function. This study showed that urinary 8-oxoGsn levels were negatively associated with gait speed and grip strength and positively correlated with the repeated chair stand test results. Based on previous literature, age, sex, lifestyles, and comorbidities, which were considered to have effects on physical function and oxidative stress, were included as confounders (6). After adjusting for potential confounders, high urinary 8-oxoGsn levels were independently associated with poor physical performance.

\section{Oxidative Stress and Cognitive Function}

Although amyloid-b plaques and neurofibrillary tangles have been considered the main cause of $\mathrm{AD}$ pathogenesis until now, many studies have proposed that oxidative damage is a key component. The brain is highly susceptible to oxidative damage because of the following four reasons: (1) the brain has a high rate of oxygen consumption; (2) the brain contains high levels of phospholipids and polyunsaturated fatty acids (29); (3) the high levels of transition metals (such as iron and copper) in the brain can catalyze the continual production of free radicals (30); and (4) the brain has low levels of antioxidant enzymes compared with other organs (31). When the level of ROS in the cell exceeds the defense ability of the cell, it undergoes various changes, including modifications of proteins, damage to lipids and DNA, and altered intrinsic properties of biomolecules, which may ultimately lead to cell death. Thus, oxidative stressmediated biomolecular damage is extensively reported in $\mathrm{AD}$, which suggests the critical role that oxidative stress plays in the development of cognitive impairment.

In 1999, Professor Akihiko Nunomura examined the concentration of 8-oxodGsn/8-oxoGsn in the brain tissue of patients with $\mathrm{AD}$ and found that oxidized nucleosides are not mainly distributed in the mitochondrial DNA with high oxidation levels but predominantly occur in the cytoplasmic RNA. This demonstrates that RNA is a major site of nucleic acid oxidative damage and that RNA oxidation is a prominent feature of vulnerable neurons in $\mathrm{AD}$ (32). In 2003, Abe et al. found a significant five-fold increase in 8-oxoGsn concentrations in the cerebrospinal fluid of patients with $\mathrm{AD}$ compared with healthy controls and that 8-oxoGsn concentrations in the cerebrospinal fluid decreased significantly with the duration of disease and progression of cognitive dysfunction (33). A previous study showed increased RNA oxidative damage in the brains of patients with mild cognitive impairment (34). The results of these in vivo studies indicate that an increase in 8-oxoGsn and oxidative RNA damage may play a role in the early stages of $\mathrm{AD}$ development. Studies on the relationship between oxidative damage and pathological changes in $\mathrm{AD}$ revealed that RNA oxidative damage precedes the formation of amyloid- $\beta$ or tau pathology (35).

In our previous studies, we found increased amounts of 8oxoGsn in the hippocampi of senescence-accelerated SAMP8 mice, which exhibited early aging syndromes and declining learning and memory compared with age-matched control mice (36). Furthermore, increased amounts of 8-oxoGsn in RNA were also observed in the hippocampi of patients with $\mathrm{AD}$ (36). Notably, we found that increased 8-oxoGsn levels in the RNA of mammals induce the aggregation of amyloid $\beta$ peptides in cells expressing amyloid precursor protein (37). In previous studies, we revealed that both the 8-oxoGsn content in the brain RNA and urine increased more rapidly in elderly mice, rats, and monkeys, indicating that urinary 8 -oxoGsn is a representative marker of oxidative RNA lesions and a biomarker for the sensitive measurement of oxidative stress and aging (37). In this study, we explored the significance of urinary 8-oxoGsn in individuals with undiagnosed dementia with cognitive decline, a relationship that has received minimal attention so far. Results showed that urinary 8-oxoGsn levels were negatively associated with the scores on the MoCA 5-min protocol. Mild cognitive impairment (MCI) is a clinical transitional status between the cognitively normal condition and $\mathrm{AD}$. We recently found that urinary 8oxoGsn may be a useful indicator for early screening for MCI in frail patients with cardiovascular disease (38). Therefore, it is a promising indicator for the early identification of cognitive impairment and needs to be explored in the future.

\section{Limitations}

This study had some limitations. First, although the results revealed significant associations between oxidative stress biomarkers and physical and cognitive impairments, the crosssectional approach limits our ability to make causal inferences. Therefore, future high-quality prospective research is warranted to confirm these findings. Second, we used a single biomarker of oxidative stress, and some other oxidative stress indicators were not included in the comparisons. Third, the urinary biomarker used in this study is still under scientific scrutiny, and the reference value range of this marker has not yet been established. Therefore, the tertile method was applied in this study to define the high, middle, and low levels, which limits the interpretation of the results and their clinical applicability.

\section{Conclusions}

Our results suggested a strong relationship between urinary 8-oxoGsn, a biomarker of oxidative damage to RNA, and physical and cognitive performance. Elevated urinary 8-oxoGsn levels are independently associated with cognitive impairment and physical decline. Furthermore, urine samples are easy to obtain, and urinary 8-oxoGsn is stable and easy to store, making it a promising indicator for implementation among community-dwelling older adults. Given the common biological mechanism of oxidative stress in physical decline and cognitive impairment, this study provides an attractive target for the early identification of decline in intrinsic abilities using a biomarker. The results of this study have important implications for the prevention of cognitive and physical decline in the 
elderly and the development of treatment strategies to promote healthy aging. In the future, cohort studies can be used to observe the causal relationship and degree of influence of this urinary indicator on cognitive and physical function decline; this indicator can be used to observe the efficacy of anti-oxidative stress treatment.

\section{DATA AVAILABILITY STATEMENT}

The original contributions presented in the study are included in the article/Supplementary Material, further inquiries can be directed to the corresponding author/s.

\section{ETHICS STATEMENT}

The study protocol was approved by the Research Ethics Committee of Beijing Hospital (2019BJYYEC-054-02), and the study was performed in accordance with the principles of the Declaration of Helsinki. The patients/participants provided their written informed consent to participate in this study.

\section{REFERENCES}

1. Rudnicka E, Napierala P, Podfigurna A, Meczekalski B, Smolarczyk R, Grymowicz M. The World Health Organization (WHO) approach to healthy ageing. Maturitas. (2020) 139:6-11. doi: 10.1016/j.maturitas.2020.05.018

2. Robertson DA, Savva GM, Kenny RA. Frailty and cognitive impairmenta review of the evidence and causal mechanisms. Ageing Res Rev. (2013) 12:840-51. doi: 10.1016/j.arr.2013.06.004

3. Baumann CW, Kwak D, Liu HM, Thompson LV. Age-induced oxidative stress: how does it influence skeletal muscle quantity and quality? J Appl Physiol. (1985) 2016;121:1047-52. doi: 10.1152/japplphysiol.00321.2016

4. Gomes MJ, Martinez PF, Pagan LU, Damatto RL, Cezar MDM, Lima ARR, et al. Skeletal muscle aging: influence of oxidative stress and physical exercise. Oncotarget. (2017) 8:20428-40. doi: 10.18632/oncotarget.14670

5. Can B, Kara O, Kizilarslanoglu MC, Arik G, Aycicek GS, Sumer F, et al. Serum markers of inflammation and oxidative stress in sarcopenia. Aging Clin Exp Res. (2017) 29:745-52. doi: 10.1007/s40520-016-0626-2

6. Liu CK, Lyass A, Larson MG, Massaro JM, Wang N, D’Agostino RB, et al. Biomarkers of oxidative stress are associated with frailty: the framingham offspring study. Age (Dordr). (2016) 38:1. doi: 10.1007/s11357-015-9864-Z

7. Tonnies E, Trushina E. Oxidative stress, synaptic dysfunction, and Alzheimer's disease. J Alzheimers Dis. (2017) 57:1105-21. doi: 10.3233/JAD-161088

8. Butterfield DA, Halliwell B. Oxidative stress, dysfunctional glucose metabolism and Alzheimer disease. Nat Rev Neurosci. (2019) 20:148-60. doi: 10.1038/s41583-019-0132-6

9. Schrag M, Mueller C, Zabel M, Crofton A, Kirsch WM, Ghribi $\mathrm{O}$, et al. Oxidative stress in blood in Alzheimer's disease and mild cognitive impairment: a meta-analysis. Neurobiol Dis. (2013) 59:100-10. doi: 10.1016/j.nbd.2013.07.005

10. Mecocci P, MacGarvey U, Kaufman AE, Koontz D, Shoffner JM, Wallace DC, et al. Oxidative damage to mitochondrial DNA shows marked age-dependent increases in human brain. Ann Neurol. (1993) 34:60916. doi: 10.1002/ana.410340416

11. Rinaldi P, Polidori MC, Metastasio A, Mariani E, Mattioli P, Cherubini A, et al. Plasma antioxidants are similarly depleted in mild cognitive impairment and in Alzheimer's disease. Neurobiol Aging. (2003) 24:9159. doi: 10.1016/S0197-4580(03)00031-9

12. Mao YH, Weng QH, Xu LN, Li XY, Zhao B, Sun Y, et al. Levels of 8oxo-dGsn and 8-oxo-Gsn in random urine are consistent with $24 \mathrm{~h}$ urine in healthy subjects and patients with renal disease. Free Radic Res. (2017) 51:616-21. doi: 10.1080/10715762.2017.1346249

\section{AUTHOR CONTRIBUTIONS}

J-PC and SJ: conceptualization and design, writing-review \& editing, and supervision. SJ: methodology, writing-original draft, and formal analysis. JC, L-QZ, ZL, YZ, and YS: acquisition of subjects and data. J-PC and JC: resources and project administration. All authors contributed to the article and approved the submitted version.

\section{FUNDING}

This work was supported by the National Key R\&D Program of China [2018YFC2000301 and 2020YFC2002700] and CAMS Innovation Fund for Medical Sciences [2021-1-I2M-1-050].

\section{SUPPLEMENTARY MATERIAL}

The Supplementary Material for this article can be found online at: https://www.frontiersin.org/articles/10.3389/fmed. 2022.816822/full\#supplementary-material

13. Jacob KD, Noren Hooten N, Trzeciak AR, Evans MK. Markers of oxidant stress that are clinically relevant in aging and age-related disease. Mech Ageing Dev. (2013) 134:139-57. doi: 10.1016/j.mad.2013.02.008

14. Gan W, Nie B, Shi F, Xu XM, Qian JC, Takagi Y, et al. Age-dependent increases in the oxidative damage of DNA, RNA, and their metabolites in normal and senescence-accelerated mice analyzed by LC-MS/MS: urinary 8oxoguanosine as a novel biomarker of aging. Free Radic Biol Med. (2012) 52:1700-7. doi: 10.1016/j.freeradbiomed.2012.02.016

15. Henriksen T, Hillestrøm PR, Poulsen HE, Weimann A. Automated method for the direct analysis of 8-oxo-guanosine and 8-oxo-2'deoxyguanosine in human urine using ultraperformance liquid chromatography and tandem mass spectrometry. Free Radic Biol Med. (2009) 47:629-35. doi: 10.1016/j.freeradbiomed.2009.06.002

16. Chen LK, Woo J, Assantachai P, Auyeung TW, Chou MY, Iijima K, et al. Asian working group for sarcopenia: 2019 consensus update on sarcopenia diagnosis and treatment. J Am Med Dir Assoc. (2020) 21:300-7 e2. doi: 10.1016/j.jamda.2019.12.012

17. Wong A, Nyenhuis D, Black SE, Law LS, Lo ES, Kwan PW, et al. Montreal Cognitive Assessment 5-min protocol is a brief, valid, reliable, and feasible cognitive screen for telephone administration. Stroke. (2015) 46:1059-64. doi: 10.1161/STROKEAHA.114.007253

18. Wong A, Xiong YY, Kwan PW, Chan AY, Lam WW, Wang K, et al. The validity, reliability and clinical utility of the Hong Kong Montreal Cognitive Assessment (HK-MoCA) in patients with cerebral small vessel disease. Dement Geriatr Cogn Disord. (2009) 28:81-7. doi: 10.1159/000 232589

19. Liew TM. The optimal short version of montreal cognitive assessment in diagnosing mild cognitive impairment and dementia. J Am Med Dir Assoc. (2019) 20:1055 e1-e8. doi: 10.1016/j.jamda.2019.02.004

20. Powers SK, Smuder AJ, Judge AR. Oxidative stress and disuse muscle atrophy: cause or consequence? Curr Opin Clin Nutr Metab Care. (2012) 15:2405. doi: 10.1097/MCO.0b013e328352b4c2

21. Jang YC, Van Remmen H. Age-associated alterations of the neuromuscular junction. Exp Gerontol. (2011) 46:193-8. doi: 10.1016/j.exger.2010.08.029

22. Bellinger AM, Mongillo M, Marks AR. Stressed out: the skeletal muscle ryanodine receptor as a target of stress. J Clin Invest. (2008) 118:44553. doi: 10.1172/JCI34006

23. D’Antona G, Pellegrino MA, Adami R, Rossi R, Carlizzi CN, Canepari $\mathrm{M}$, et al. The effect of ageing and immobilization on structure and function of human skeletal muscle fibres. J Physiol. (2003) 552:499511. doi: 10.1113/jphysiol.2003.046276 
24. Afilalo J. Conceptual models of frailty: The sarcopenia phenotype. Can J Cardiol. (2016) 32:1051-5. doi: 10.1016/j.cjca.2016.05.017

25. Soysal P, Isik AT, Carvalho AF, Fernandes BS, Solmi M, Schofield P, et al. Oxidative stress and frailty: A systematic review and synthesis of the best evidence. Maturitas. (2017) 99:66-72. doi: 10.1016/j.maturitas.2017.01.006

26. Howard C, Ferrucci L, Sun K, Fried LP, Walston J, Varadhan R, et al. Oxidative protein damage is associated with poor grip strength among older women living in the community. J Appl Physiol. (1985) (2007) 103:1720. doi: 10.1152/japplphysiol.00133.2007

27. Liang YD, Liu Q, Du MH, Liu Z, Yao SM, Zheng PP, et al. Urinary 8oxo-7,8-dihydroguanosine as a potential biomarker of frailty for elderly patients with cardiovascular disease. Free Radic Biol Med. (2020) 152:24854. doi: 10.1016/j.freeradbiomed.2020.03.011

28. Jiang S, Kang L, Zhu M, Zhang X, Wang C, Cai J, et al. A potential urinary biomarker to determine frailty status among older adults. Arch Gerontol Geriatr. (2020) 88:104038. doi: 10.1016/j.archger.2020.104038

29. Singh A, Kukreti R, Saso L, Kukreti S. Oxidative Stress: A key modulator in neurodegenerative diseases. Molecules. (2019) 24: doi: 10.3390/molecules24081583

30. Greenough MA, Camakaris J, Bush AI. Metal dyshomeostasis and oxidative stress in Alzheimer's disease. Neurochem Int. (2013) 62:54055. doi: 10.1016/j.neuint.2012.08.014

31. Mecocci P, Boccardi V, Cecchetti R, Bastiani P, Scamosci M, Ruggiero $\mathrm{C}$, et al. A long journey into aging, brain aging, and Alzheimer's disease following the oxidative stress tracks. J Alzheimers Dis. (2018) 62:131935. doi: 10.3233/JAD-170732

32. Nunomura A, Perry G, Pappolla MA, Wade R, Hirai K, Chiba S, et al. RNA oxidation is a prominent feature of vulnerable neurons in Alzheimer's disease. J Neurosci. (1999) 19:1959-64. doi: 10.1523/JNEUROSCI.19-06-01959.1999

33. Abe $\mathrm{T}$, Tohgi $\mathrm{H}$, Isobe $\mathrm{C}$, Murata $\mathrm{T}$, Sato C. Remarkable increase in the concentration of 8-hydroxyguanosine in cerebrospinal fluid from patients with Alzheimer's disease. J Neurosci Res. (2002) 70:447-50. doi: 10.1002/jnr.10349

34. Lovell MA, Markesbery WR. Oxidatively modified RNA in mild cognitive impairment. Neurobiol Dis. (2008) 29:169-75. doi: 10.1016/j.nbd.2007.07.030
35. Nunomura A, Perry G, Aliev G, Hirai K, Takeda A, Balraj EK, et al. Oxidative damage is the earliest event in Alzheimer disease. J Neuropathol Exp Neurol. (2001) 60:759-67. doi: 10.1093/jnen/60.8.759

36. Song XN, Zhang LQ, Liu DG, Lin J, Zheng JD, Dai DP, et al. Oxidative damage to RNA and expression patterns of MTH1 in the hippocampi of senescence-accelerated SAMP8 mice and Alzheimer's disease patients. Neurochem Res. (2011) 36:1558-65. doi: 10.1007/s11064-0110484-4

37. Dai DP, Gan W, Hayakawa H, Zhu JL, Zhang XQ, Hu GX, et al. Transcriptional mutagenesis mediated by 8-oxoG induces translational errors in mammalian cells. Proc Natl Acad Sci U S A. (2018) 115:421822. doi: $10.1073 /$ pnas. 1718363115

38. Yao SM, Zheng PP, He W, Cai JP, Wang H, Yang JF. Urinary 8OxoGsn as a potential indicator of mild cognitive impairment in frail patients with cardiovascular disease. Front Aging Neurosci. (2021) 13:672548. doi: 10.3389/fnagi.2021.672548

Conflict of Interest: The authors declare that the research was conducted in the absence of any commercial or financial relationships that could be construed as a potential conflict of interest.

Publisher's Note: All claims expressed in this article are solely those of the authors and do not necessarily represent those of their affiliated organizations, or those of the publisher, the editors and the reviewers. Any product that may be evaluated in this article, or claim that may be made by its manufacturer, is not guaranteed or endorsed by the publisher.

Copyright (C) 2022 Jiang, Cui, Zhang, Liu, Zhang, Shi and Cai. This is an open-access article distributed under the terms of the Creative Commons Attribution License (CC $B Y)$. The use, distribution or reproduction in other forums is permitted, provided the original author(s) and the copyright owner(s) are credited and that the original publication in this journal is cited, in accordance with accepted academic practice. No use, distribution or reproduction is permitted which does not comply with these terms. 